\title{
Peak Power Effect on Skin Rejuvenation Using IPL: Lumecca IPL Evaluation
}

\author{
E. V. Ross, Michael Kreindel \\ Irvine, CA, USA \\ Email: kreindel66@gmail.com
}

Received 4 April 2016; accepted 13 May 2016; published 18 May 2016

Copyright (C) 2016 by authors and Scientific Research Publishing Inc.

This work is licensed under the Creative Commons Attribution International License (CC BY). http://creativecommons.org/licenses/by/4.0/

(c) ()

\begin{abstract}
A high peak power IPL system (Lumecca) was tested to determine the correlation between a high peak power and the successful treatment of pigmented and vascular lesions. Short pulse duration in the millisecond range and high peak power of $3.3 \mathrm{~kW} / \mathrm{cm}^{2}$ enabled selective and effective destruction, not only of pigment, but also of vessels in a comparable manner to a pulsed dye laser. Only one treatment session at a low fluence $\left(8-16 \mathrm{~J} / \mathrm{cm}^{2}\right)$ was sufficient to achieve the desired results.
\end{abstract}

\section{Keywords}

Intense Pulsed Light, Peak Power Effect, Skin Rejuvenation

\section{Introduction}

Intense Pulsed Light (IPL) technology has become a common tool in medical aesthetic practices for skin rejuvenation. The popularity of IPL technology has increased because of its simplicity, low cost and versatility, allowing for treatment of a variety of vascular and pigmented lesions. The broad spectrum of IPL covers the visible and near infrared spectrum and can be easily adjusted for specific applications by using filters and optimizing lamp parameters. The spectrum of IPL may be used for treatment of pigmented lesions with different levels of aggression. It includes multiple peaks of hemoglobin absorption, and penetrates into the skin down to a few millimeters at longer wavelengths.

The other advantage of IPL is the variability of the pulse width in a relatively broad range from a few milliseconds up to a few hundred milliseconds. Energy can be delivered in a single pulse or in a train of pulses.

Even the first IPL had multiple filters and a sophisticated pulse structure [1]. Future efforts were directed to the optimization of broad spectrum filtering, trying to reach the efficiency of Pulse Dye Laser (PDL) for treatment of superficial vascular lesions [2]. Treatment results were comparable but at a significantly higher fluence 
than with PDL. These results can be interpreted in two ways: Either the IPL has less treatment selectivity, or the peak power of the IPL is lower than that of the laser.

The treatment of vascular and pigmented lesions is based on principles of selective photothermolysis suggested by Anderson and Parish [3]: a. Light penetration depth should be high enough to reach the treatment target; b. Light absorption by the treatment target should be higher than that by surrounding tissue; c. Light should be delivered in pulses with pulse duration that is shorter than the Thermal Relaxation Time (TRT) of the treatment target.

The authors of [2] tried to demonstrate that long pulse durations of up to $400 \mathrm{msec}$ can be as effective as a short pulse, but this concept has proven relevant only in particular scenarios where the "heater" and "real" targets are dimensionally optimized for "very" long pulsed applications. On the contrary, home-use IPLs with submillisecond pulses demonstrate reasonable efficiency using fluences of $3-5 \mathrm{~J} / \mathrm{cm}^{2}$ [4].

Higher peak powers allow for the following potential treatment benefits: a. Coagulation temperature in the target can be reached at a lower fluence; b. Higher selectivity; c. Smaller targets can be treated.

This article is intended to demonstrate efficiency of the IPL device that is designed to provide the highest peak power and shorter pulse duration for treatment of vascular and pigmented lesions.

\section{Materials and Methods}

The Lumecca IPL device (InModeMD Ltd., Yokneam, Israel) was used for treatment of vascular and pigmented lesions. The Lumecca IPL device has a peak power of $3.3 \mathrm{~kW} / \mathrm{cm}^{2}$ and $9.9 \mathrm{~kW} / 3 \mathrm{~cm}^{2}$ light-guide area. That combination allows for effective treatment with a low fluence of $10 \mathrm{~J} / \mathrm{cm}^{2}$ at a pulse duration of only $3 \mathrm{msec}$. Such pulse durations correspond to the TRT of vessels with diameters of $70 \mu \mathrm{m}$. For comparison, a pulse width of $20 \mathrm{msec}$ corresponds to a TRT of a vessel with diameter of $18 \mu \mathrm{m}$ only.

Patients were treated with a single treatment, using fluence of $8-16 \mathrm{~J} / \mathrm{cm}^{2}$ and filter of $515 \mathrm{~nm}$ and were followed-up after four weeks.

\section{Results}

All patients demonstrated significant improvement of skin discoloration related to vascular and pigmented lesions. The treatment pigment results were achieved with fluences as low as $8 \mathrm{~J} / \mathrm{cm}^{2}$. Typical outcome for pigmentation with Lumecca treatment is shown in Figure 1.

Sometimes the Lumecca treatment was accompanied by the same minor purpura that is observed typically with the pulsed dye laser at such a low fluence level. That finding indicates a similar level of selectivity for vascular lesions.

To avoid purpura, the Lumecca $580 \mathrm{~nm}$ has been used to reduce aggressiveness of a treatment, or the longer pulse mode can be applied with the $515 \mathrm{~nm}$ handpiece.

For larger vessels with the $515 \mathrm{~nm}$ filtered handpiece, higher fluences of $12-14 \mathrm{~J} / \mathrm{cm}^{2}$ were used in combination with stronger skin cooling (Figure 2). The ability of the device to provide vessel closure at such low fluences can be explained by two reasons: a. The peak power of the Lumecca is higher than for most other IPL devices. b. High peak power introduced into the lamp during the pulse results in a spectrum shift to the shorter wavelengths and increase of optical energy in the range of $500-600 \mathrm{~nm}$ where absorption of the melanin and hemoglobin is maximal.

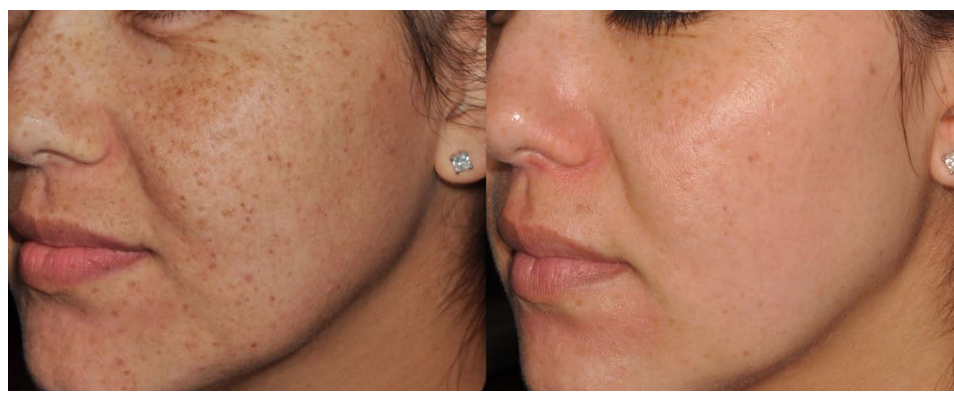

Figure 1. Before (left) and 4 weeks after (right) single Lumecca $515 \mathrm{~nm}$ treatment at $10 \mathrm{~J} / \mathrm{cm}^{2}$. 


\section{Discussion}

Theoretical considerations indicate that pulse width and its relation with target- specific thermal relaxation times (TRT) is one of the critical elements of selective photothermolysis [5].

$T R T=\frac{d^{2}}{A \alpha}$, where $\mathrm{d}$ is size of the treated target, A geometrical factor $(A=16$ for cylindrical target and $A=4$ for planar target), $\alpha$ is diffusivity of tissue and can be estimated as one of water $\left(\alpha \sim 10^{-7} \mathrm{~m}^{2} / \mathrm{sec}\right)$.

If pulse width is longer than TRT of the target, a significant part of the energy is dissipated from the target to the surrounding tissue. Figure 3 shows temperature distribution for the pulse with duration of 0.3 of the TRT and $3 \times$ longer than the TRT. It is apparent that at the same applied energy, the peak target temperature can be $2 \times$ that associated with the longer pulse. Consequently, half of the energy is sufficient to reach coagulation temperature.

In order to destroy single melanosomes, the nano- or picosecond pulse durations are optimal, whereas IPL and other millisecond devices are more likely to heat the entire dermal-epidermal junction layer. Shorter pulses increase selectivity such that even so called "low contrast” lesions can be treated. Shorter pulses also allow treatment of "early-stage" less pigmented lesions, at times even when they are not visible by the naked eye. Longer pulse injuries are less selective and more discretion must be used by the operator to avoid higher risk of side effects.

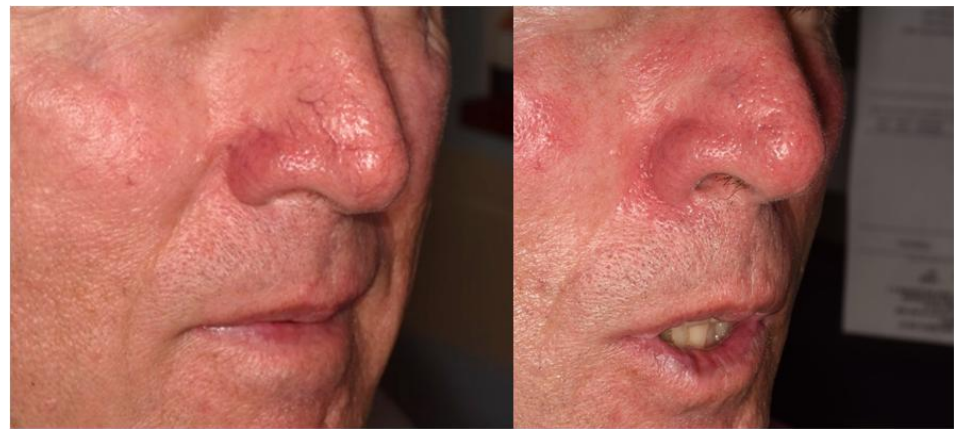

Figure 2. Before (left) and immediately after (right) single Lumecca $515 \mathrm{~nm}$ treatment at 14 $\mathrm{J} / \mathrm{cm}^{2}$.

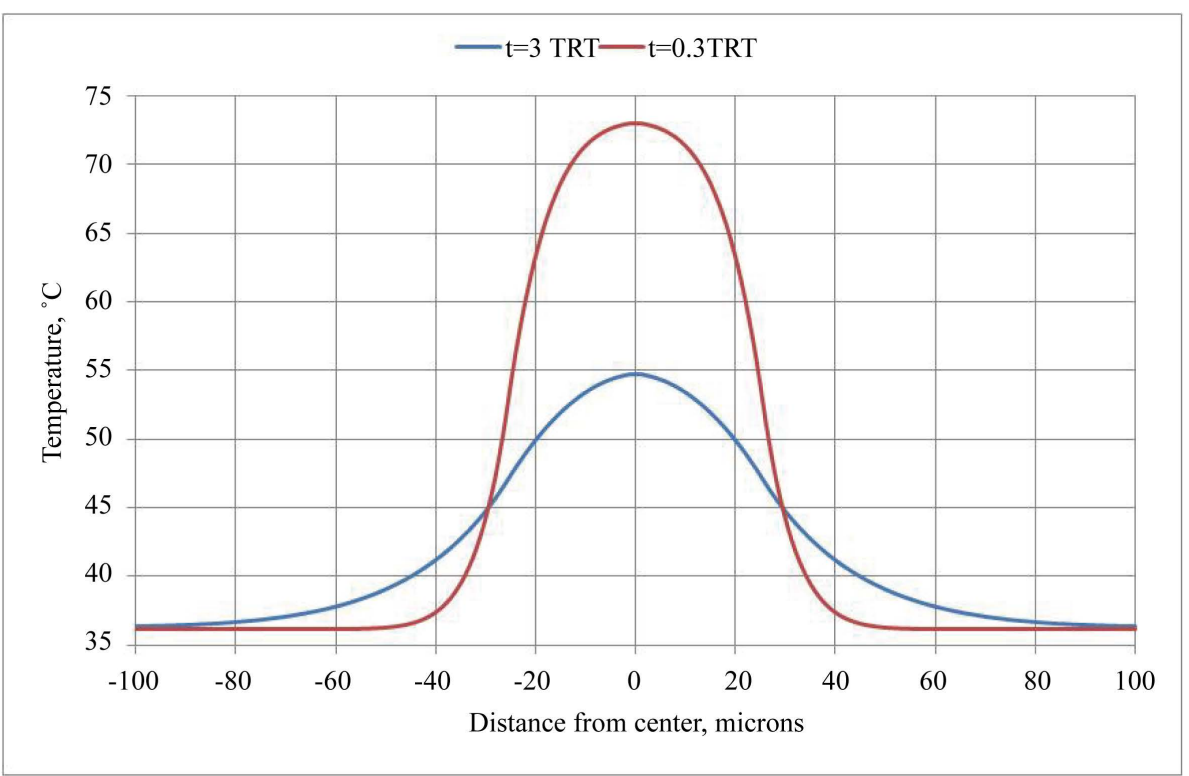

Figure 3. Temperature of 50 micron cylindrical object heated with pulse width three times longer than TRT and 3 times shorter than TRT. 


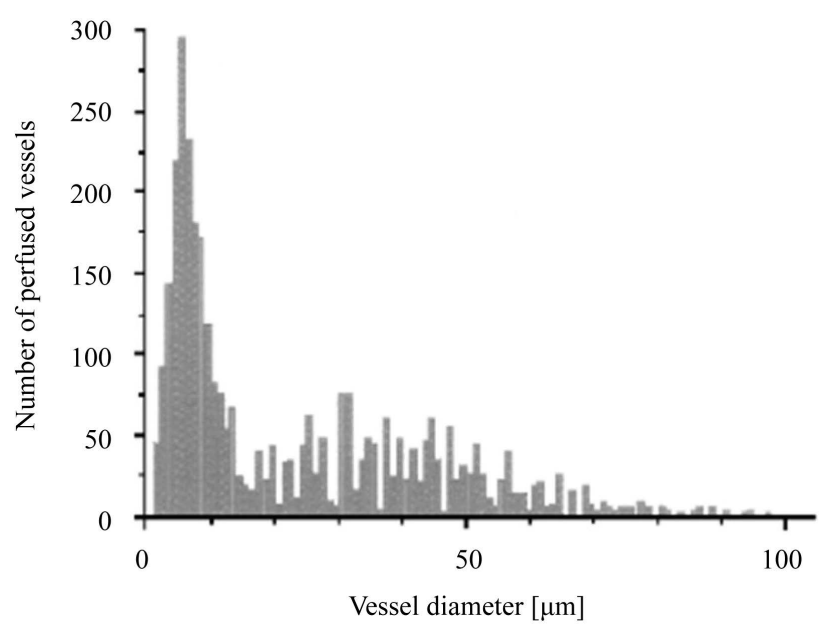

Figure 4. Number of perfused blood vessels versus vessel diameter [7].

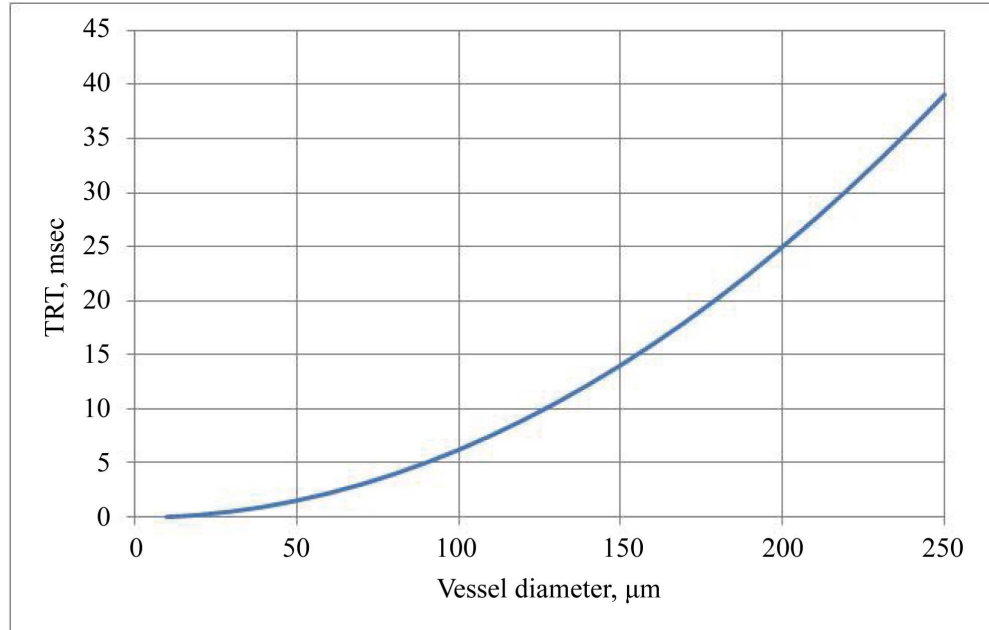

Figure 5. TRT versus vessel diameter.

The relative number of blood vessels in a body is strongly related to their size. One study [6] analyzed dimensions of blood vessels in a bat's wing and showed that the number of venules exceeded the number of small veins by fifteen times and the number of post-capillary venules was five times higher than number of venules.

Authors of another study [7] measured a number of perfused vessels in human skin. They ranged $5-12 \mu \mathrm{m}$ on the lower end (for capillaries) and then up to $250 \mu \mathrm{m}$ vessels. Measurements were performed using intravital microscopy. Results of measurements are shown in Figure 4.

This data demonstrated that the ability to treat smaller vessels, presumably with shorter pulses, exponentially increased the number of vessels that can be targeted by a device.

Figure 5 shows correlation of blood vessel TRT with vessel diameter.

We can see that for treatment of vessels smaller than $100 \mu \mathrm{m}$ the pulse should be shorter than $7 \mathrm{msec}$.

\section{Conclusion}

Single treatment with Lumecca provides significant improvement for majority of patients for both pigmented and vascular lesions. The high peak power of Lumecca makes its effect on vascular lesions comparable to PDL.

\section{References}

[1] Goldman, M.P., Eckhouse, S., ESC Medical Systems, and LTD Photoderm VL Cooperative Study Group (1996) Pho- 
tothermal Sclerosis of Leg Veins. Dermatologic Surgery, 22, 323-330. http://dx.doi.org/10.1111/j.1524-4725.1996.tb00325.x

[2] Weiss, R.A., Ross, E.V., Tanghetti, E.A., Vasily, D.B., Childs, J.J., Smirnov, M.Z. and Altshuler, G.B. (2011) Characterization of an Optimized Light Source and Comparison to Pulsed Dye Laser for Superficial and Deep Vessel Clearance. Lasers in Surgery and Medicine, 43, 92-98. http://dx.doi.org/10.1002/lsm.21032

[3] Anderson, R.R. and Parrish, J.A. (1983) Selective Photothermolysis: Precise Microsurgery by Selective Absorption of Pulsed Radiation. Science, 220, 524-527. http://dx.doi.org/10.1126/science.6836297

[4] Town, G. and Ash, C. (2010) Are Home-Use Intense Pulsed Light (IPL) Devices Safe? Lasers in Medical Sciences, 25, 773-780.

[5] van Gemert, M.J. and Welch, A.J. (1989) Time Constants in Thermal Laser Medicine. Lasers in Surgery and Medicine, 9, 405-421. http://dx.doi.org/10.1002/lsm.1900090414

[6] Wiedeman, M.P. (1963) Dimensions of Blood Vessels from Distributing Artery to Collecting Vein. Circulation Research, XLL, 375-378.

[7] Babilas, P., Shafirstein, G., Bäumler, W., Baier, J., Landthaler, M., Szeimies, R.-M. and Abels, C. (2005) Selective Photothermolysis of Blood Vessels Following Flashlamp-Pumped Pulsed Dye Laser Irradiation: In Vivo Results and Mathematical Modelling Are in Agreement. Journal of Investigative Dermatology, 125, 343-352; 